\title{
COMPARACIÓN DEL HYPER-CVAD CON UN RÉGIMEN INSTITUCIONAL EN EL TRATAMIENTO DE LA LEUCEMIA LINFOBLÁSTICA AGUDA DEL ADULTO EN UN HOSPITAL DE MÉXICO
}

\author{
Christian Omar Ramos-Peñafiel1,2,a , Álvaro Cabrera-García ${ }^{1,2, a}$, Etta Rozen-Fuller1,2,a, \\ Guadalupe González-León 1,2,a, Carolina Balderas ${ }^{1,2, a}$, Juan Julio Kassack-Ipiña ${ }^{1,2, a}$, \\ Humberto Castellanos-Sinco ${ }^{1,2, a}$, Carlos Martínez-Murillo ${ }^{1,2, a}$, Efreen Montaño-Figueroa ${ }^{1,2, a}$, \\ Adolfo Martínez-Tovar ${ }^{1, b}$, Irma Olarte-Carrillo ${ }^{1, c}$, Adrián Santoyo-Sánchez ${ }^{2, d}$, Juan Collazo-Jaloma1,2,a
}

\begin{abstract}
RESUMEN
Con el objetivo de evaluar la mortalidad y toxicidad del protocolo Hyper-CVAD utilizado como primera línea de tratamiento de la leucemia linfoblástica aguda se realizó un estudio de cohorte retrospectiva en pacientes de 40 años a menos durante marzo a septiembre de 2011 atendidos con el régimen Hyper-CVAD. La mortalidad y toxicidad se comparó con los resultados de los pacientes atendidos con el régimen institucional HGMLAL07 entre 2009 a 2012. Se incluyeron 18 pacientes, la mediana de edad fue de 26 años. Tanto las remisiones completas $(67,7 \%$ frente a $81,9 \%)$ como la supervivencia a un año $(40 \%$ frente a $62 \%$ ) y 2 años (18\% frente a $34 \%)$ fueron menores con el régimen Hyper-CVAD. Al seleccionar exclusivamente pacientes menores de 35 años, la eficacia de Hyper-CVAD también fue menor. Según esta experiencia y debido a su alto costo y toxicidad, el régimen Hyper-CVAD debe de limitarse a aquellos pacientes con leucemias refractarias o en recaída.
\end{abstract}

Palabras clave: Leucemia linfoblástica; Protocolos de quimioterapia combinada antineoplásica; Adulto (fuente DeCS BIREME).

\section{COMPARISON OF THE HYPER-CVAD WITH AN INSTITUTIONAL REGIMEN FOR THE TREATMENT OF ACUTE LYMPHOBLASTIC LEUKEMIA IN ADULTS IN A HOSPITAL OF MEXICO}

\begin{abstract}
In order to assess the mortality and toxicity of the Hyper-CVAD protocol used as first-line treatment of acute lymphoblastic leukemia, a retrospective cohort study was performed in patients less than 40 years of age from March to September 2011 treated with Hyper-CVAD regimen. Mortality and toxicity was compared with the results of patients treated with the institutional HGMLAL07 regimen between 2009-2012. 18 patients were included; the median age was 26 years old. Complete remissions $(67.7 \%$ versus $81.9 \%)$ as well as one-year ( $40 \%$ versus $62 \%)$ and 2 year survival rates $(18 \%$ versus $34 \%$ ) were lower with the Hyper-CVAD regimen. By selecting only patients younger than 35 years, the effectiveness of Hyper-CVAD was also lower. In our experience and because of its high cost and toxicity, the Hyper-CVAD regimen should be limited to patients with relapsed or refractory leukemia.
\end{abstract}

Key words: Leukemia, lymphoblastic; Antineoplastic combined chemotherapy protocols; Adult (source: MeSH, NLM).

\footnotetext{
Hospital General de México. Distrito Federal, México

Facultad de Medicina, Universidad Nacional Autónoma de México. Distrito Federal, México

Médico cirujano; ${ }^{\mathrm{b}}$ Doctor en Genética y Biología Molecular; ${ }^{\mathrm{C}}$ doctor en Ciencias Biológicas y de la Salud; ${ }^{\mathrm{d}}$ estudiante de Medicina. Recibido: : 06-03-14 Aprobado: 06-08-14
} 


\section{INTRODUCCIÓN}

El tratamiento de la leucemia linfoblástica aguda combina actualmente quimioterapia, inmunoterapia y blancos moleculares ${ }^{(1-3)}$. Uno de los esquemas más populares es el Hyper-CVAD (4). Desarrollado inicialmente para recaídas ${ }^{(5)}$ y actualmente como uso de primera línea, el Hyper-CVAD ha sufrido diversas modificaciones siendo las principales el uso de anticuerpos monoclonales (rituximab) (6) e inhibidores de cinasa de tirosina (dasatinib) (7). En el reporte original, Kantarjian et al. registraron una tasa de remisiones completas del $92 \%$, con una mortalidad en inducción del $5 \%{ }^{(8)}$.

Desde entonces, diversos autores han adaptado este régimen pero no con el éxito del Anderson Cancer Center. Xu et al. en su reporte de 38 casos, registraron una tasa de remisiones del $73,6 \%$ con una supervivencia a dos años del 82,9\% ${ }^{(9)}$. En América Latina Combariza et al. evaluaron 83 pacientes tratados con HyperCVAD como primera línea de tratamiento, su tasa de remisiones completas reportadas fueron del $61 \%$ con una supervivencia global de 11,3 meses ${ }^{(10)}$. El Hospital General de México cuenta con el régimen institucional HGMLAL07 desde el año 2007, fundamentado en el protocolo GIMEMA ALL0288 ${ }^{(12)}$, debido a que la experiencia de los autores con el esquema Hyper-CVAD se limita solo a leucemias en recaída, el objetivo del estudio fue evaluar este esquema como primera línea en pacientes jóvenes y con un buen estado funcional.

\section{EL ESTUDIO}

Se realizó una cohorte retrospectiva basada en pacientes atendidos desde el 2009 al 2012 con los dos esquemas registrados como de primera línea de tratamiento (Hyper-CVAD y el protocolo institucional HGMLAL07). De forma intencionada, desde marzo de 2011, con la finalidad de comprobar la superioridad del régimen Hyper-CVAD se utilizó como esquema de elección para pacientes menores de 40 años, con un adecuado estado funcional y sin compromiso hepático o renal. Aquellos pacientes no candidatos al régimen Hyper-CVAD fueron atendidos en base del protocolo Institucional HGMLAL07. Para demostrar la superioridad del régimen Hyper-CVAD sobre la tasa de remisiones completas, se calculó el número de pacientes necesarios a tratar en base a un poder del $80 \%$, con una expectativa de éxito del régimen Hyper-CVAD del $89 \%$ (grupo 1) frente al resultado de remisiones completas ya conocido del régimen HGMLAL07 (68\%) (13). Se realizaron análisis secuenciales cada 4 meses y, en caso de que los resultados (remisiones completas) fueran inferiores que el régimen institucional, se daría por finalizado el enrolamiento y se suspendería el estudio y los pacientes subsecuentes serán atendidos con el protocolo institucional HGMLAL07.

El esquema terapéutico consiste en dos bloques: Bloque A: ciclofosfamida $300 \mathrm{mg} / \mathrm{m}^{2} \mathrm{SC}$ cada $12 \mathrm{~h}$, días 1,2 , 3; plus Mesna $1800 \mathrm{mg} / \mathrm{m}^{2}$ en perfusión continua los días 1-4; doxorrubicina $50 \mathrm{mg} / \mathrm{m}^{2}$ día +4 y vincristina $1,5 \mathrm{mg} / \mathrm{m}^{2}$ máximo $2 \mathrm{mg}$ dosis los días 4 y 11 de cada ciclo; dexametasona $40 \mathrm{mg}$ IV los días 1-4, 11-14. La quimioterapia intratecal se administró en base de dosis de citarabina $100 \mathrm{mglT}$ los días 7 de cada ciclo y metotrexate $12 \mathrm{mg}$ IT el día 2 de cada ciclo. Bloque B: metotrexato $1 \mathrm{~g} / \mathrm{m}^{2}$ $\mathrm{SC}$ el día +1 de la quimioterapia, con su rescate con ácido folínico a las $24 \mathrm{~h}$ de finalizado el metotrexato; citarabina los días +2 y +3 de cada ciclo $\left(3 \mathrm{~g} / \mathrm{m}^{2} \mathrm{SC}\right.$, a pasar en $2 \mathrm{~h}$ cada $12 \mathrm{~h}$ ). Las dosis intratecales son las mismas que en el régimen con ciclofosfamida. Los intervalos establecidos entre cada ciclo fueron de 21 días. En caso de tratarse de una LLA-Ph+ se agregó al régimen Imatinib (Gleevec@), Novartis) a dosis de $400 \mathrm{mg}$ PO los días 1-15 de cada uno de los bloques. Todos los pacientes recibieron soporte con factor estimulante de colonias de granulocitos hasta la recuperación de la neutropenia.

Para la evaluación de la respuesta al tratamiento se consideró como criterio de remisión completa, remisiones completas a la presencia de menos de $5 \%$ de Blastos en médula ósea a las cuatro semanas posteriores al tratamiento y como leucemia en recaída a la presencia de más del $5 \%$ de blastos en cualquier momento del seguimiento. Si a las cuatro semanas del tratamiento persiste una cifra mayor de $5 \%$ de blastos se considerará como leucemia refractaria. Todos los pacientes contaban con inmunofenotipo y prueba de PCR-para la búsqueda del transcrito BCR-ABL1.

Se utilizó el software SPSS versión 20.0. Para la prueba de contraste de hipótesis entre el éxito de tratamiento (remisiones completas o muerte) y el tipo de tratamiento se usó la prueba no paramétrica chi cuadrado, considerándose como significativo un valor de $p<0,05$ establecido a un $95 \%$ de intervalo de confianza. Se ha utilizado el método de Kaplan-Meier para analizar la supervivencia tras el inicio de los tratamientos y la prueba de log-rank para comparar la supervivencia entre ambos grupos.

El esquema Hyper-CVAD, al igual que el protocolo institucional HGMLAL07, se encuentran registrados y autorizados en el Hospital General de México como parte de los esquemas de primera línea. Todos los pacientes contaban con consentimiento informado de la institución para su ingreso, realización de procedimientos terapéuticos y soporte transfusional. 


\section{HALLAZGOS}

Se atendieron 18 pacientes con el esquema HyperCVAD de primera línea y se compararon con el registro histórico para un total de 90 pacientes. La mediana de edad fue menor en el grupo de Hyper-CVAD (26 años, rango de 18 a 39) en comparación con la cohorte histórica (28 años, rango de 17 a 64). La mediana de leucocitos al diagnóstico fue mayor en el grupo de Hyper-CVAD $(52,9 \times 103 / \mathrm{mcL})$, lo que condicionó un mayor número de pacientes con un riesgo alto de recaída $(77,8 \%)$ a diferencia del control histórico $(59,7 \%)$. No se registró infiltración al sistema nervioso central en el momento del diagnóstico. Las características generales de los pacientes se describen en la Tabla 1.

Tabla 1. Características generales de los pacientes

\begin{tabular}{|c|c|c|}
\hline \multirow[t]{2}{*}{ Característica } & $\begin{array}{c}\text { Hyper-CVAD } \\
(n=18)\end{array}$ & $\begin{array}{c}\text { HGMLAL07 } \\
(n=72)\end{array}$ \\
\hline & 2011 & 2009-2012 \\
\hline Edad en años* & $26(18-39)$ & $28,5(17-64)$ \\
\hline \multicolumn{3}{|l|}{ Sexo (\%) } \\
\hline Femenino & $36(50)$ & $11(61,1)$ \\
\hline Masculino & $36(50)$ & $7 \quad(38,9)$ \\
\hline \multicolumn{3}{|l|}{ Hallazgos clínicos (\%) } \\
\hline Hepatomegalia & $6(33,3)$ & $19(26,4)$ \\
\hline Esplenomegalia & $7(38,9)$ & $15(20,8)$ \\
\hline Crecimiento ganglionar & $6(33,3)$ & $24(33,3)$ \\
\hline \multicolumn{3}{|l|}{ Hallazgos de laboratorio* } \\
\hline Leucocitos ( x 103/mcL) & $52,9(0,7-709)$ & $23,6(0,3-690)$ \\
\hline Hemoglobina (g/dL) & $6,5(3,9-14)$ & $7,6(2,9-15,5)$ \\
\hline Plaquetas ( x 103/mcL) & $25(7-196)$ & $19,5(2-447)$ \\
\hline Creatinina (mg/dL) & $0,8(0,49-1,81)$ & $0,8(0,3-1,9)$ \\
\hline Urea (mg/dL) & $26,7(8,6-137)$ & $27,8(2,1-126)$ \\
\hline Ácido úrico (mg/dL) & $5,1(2,6-11,5)$ & $4,8(1,3-17)$ \\
\hline Bilirrubina (mg/dL) & $0,7(0,2-1,7)$ & $0,8(0,2-21,4)$ \\
\hline $\begin{array}{l}\text { Alanino aminotransferasa } \\
\text { (UI/L) }\end{array}$ & $38,5(9-142)$ & 38 (9-1053) \\
\hline $\begin{array}{l}\text { Aspartato } \\
\text { aminotransferasa(UI/L) }\end{array}$ & $37(13-61)$ & $34,5(11-1205)$ \\
\hline Fosfatada Alcalina (mg/dL) & $82(47-396)$ & $102(31-1199)$ \\
\hline $\begin{array}{l}\text { Deshidrogenasa láctica } \\
\text { (UI/L) }\end{array}$ & $558(113-1757)$ & $296(4,7-8541)$ \\
\hline \multicolumn{3}{|l|}{ Inmunofenotipo (\%) } \\
\hline Leucemia de precursores B & $14(77,8)$ & $66(91,7)$ \\
\hline Leucemia de precursores $T$ & $4(22,2)$ & $06(8,3)$ \\
\hline CD20 & $10(55,6)$ & $12(16,7)$ \\
\hline CD10 & $14(77,8)$ & $43(59,7)$ \\
\hline CD13 & $02(11,1)$ & $01(1,4)$ \\
\hline \multicolumn{3}{|l|}{ Leucemia $\mathrm{Ph}+(\%)$} \\
\hline Positivo & $04(22,2)$ & $04(5,6)$ \\
\hline Negativo & $14(77,8)$ & $68(94,4)$ \\
\hline \multicolumn{3}{|l|}{ Tipo de riego (\%) } \\
\hline Riesgo habitual & $04(22,2)$ & $29(40,3)$ \\
\hline Riesgo alto & $14(77,8)$ & $43(59,7)$ \\
\hline
\end{tabular}

*Mediana (rango)
En los resultados de la inducción a la remisión, del total de los 18 pacientes que iniciaron el esquema de inducción, el $67,7 \%$ (12) integró remisiones completas en comparación con un $81,9 \%$ (59) del protocolo institucional. En los dos grupos se registró un $11,1 \%$ de leucemias refractarias (2 frente a 8 casos) La mortalidad en inducción fue mayor para Hyper-CVAD con un $22,2 \%$ (4) en su mayoría asociada a neutropenia febril. A pesar de que el porcentaje de remisiones completas fue menor en el régimen HyperCVAD (81,9\% frente a $66,7 \%)$, la prueba de chi cuadrado no estableció diferencias significativas $(p=0,197)$. Del total de los 18 pacientes, 8 desarrollaron un episodio de neutropenia febril. El momento en el que se presentó más episodios de neutropenia febril fue el brazo B (92\%) seguido del brazo $A(86 \%)$ El principal germen aislado fue Staphylococcus epidermidis seguido de Escherichia coli en aparato urinario. La media de estancia hospitalaria también fue mayor para el régimen Hyper-CVAD $(37,2$ frente a 29,3 días).

De acuerdo con los resultados del seguimiento, de aquellos casos que integraron remisión, el 38,9\% (7) presentó recaída a médula ósea durante el primer año de seguimiento, en comparación al $47,2 \%$ del registro histórico sin diferencias significativas entre las recaídas y las dos estrategias terapéuticas $(p=0,603)$. Cinco de cada siete casos que se mantenían en remisión fallecieron por neutropenia febril durante los bloques secuenciales. Dos casos restantes solicitaron su modificación de tratamiento, justificaron el pedido aduciendo el tiempo prolongado de estancia hospitalaria.

La supervivencia global con el esquema Hyper-CVAD fue del 40 y $18 \%$ a un uno y dos años respectivamente. Haciendo una comparación con el registro histórico la supervivencia global fue menor tanto a un año $(40 \%$ frente a $62 \%$ ) como a 2 años de seguimiento ( $18 \%$ frente

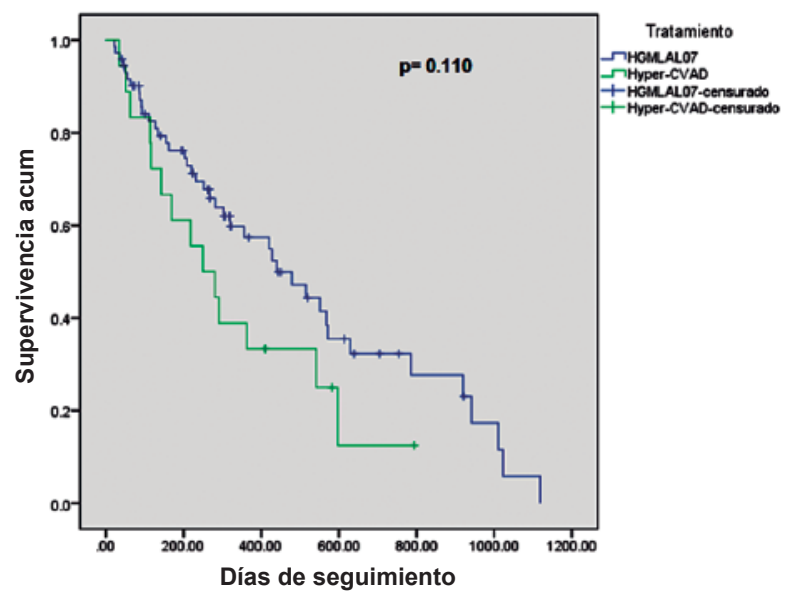

Figura 1. Supervivencia libre de la enfermedad acorde al tipo de régimen de tratamiento 


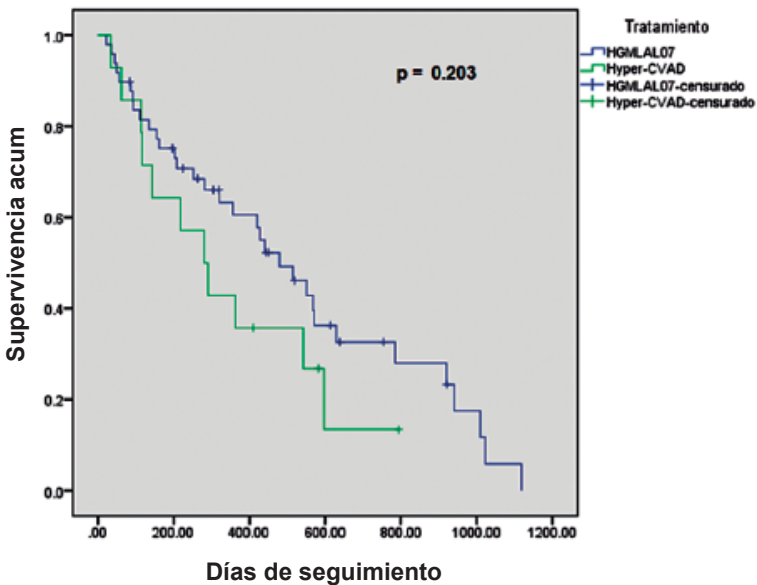

Figura 2. Supervivencia libre de la enfermedad según al tratamiento en pacientes jóvenes

a $34 \%$ ), pero sin ser significativa $(p=0,110)$ (Figura 1). La supervivencia libre de enfermedad tampoco evidenció diferencias significativas (log-rank $p=0,460$ ).

El análisis multivariado, que incluyó factores ya establecidos en la literatura para el riesgo de recaída (edad > 35 años, cuenta de leucocitos al diagnóstico $>30 \times 103 / \mathrm{mcL}$, riesgo alto, presencia de cromosoma Philadelphia o ausencia de respuesta a las 4 semanas) en conjunto con el régimen Hyper-CVAD, no mostraron valores significativos. Los resultados del análisis multivariado se presentan en la Tabla 2.

Al analizar exclusivamente a pacientes menores de 35 años en ambas cohortes, los resultados fueron semejantes, se observó una tasa mayor de remisiones completas con el protocolo institucional en comparación con Hyper-CVAD (87,8\% frente a $78,6 \%$.), y la mortalidad en inducción fue menor ( $0 \%$ frente a 14,3\%). La curva de supervivencia también fue menor en el brazo de Hyper-CVAD (281 frente a 479 días), pero sin presentar una diferencia estadística $(p=0,203)$ (Figura 2$)$.

Tabla 2. Análisis de regresión de Cox de las diferentes variables y su impacto sobre la supervivencia

\begin{tabular}{lccc}
\hline \multicolumn{1}{c}{ Variables } & HR & (IC 95\%) & $\begin{array}{c}\text { Valor } \\
\text { de } \boldsymbol{p}\end{array}$ \\
\hline Edad mayor de 35 años & 1,19 & $(0,59-2,37)$ & 0,621 \\
$\begin{array}{l}\text { Leucocitos }>30 \times 10^{3} / \mathrm{mcL} \\
\begin{array}{l}\text { Resultado positivo para } \\
\text { BCR-ABL1 }\end{array}\end{array}$ & 1,29 & $(0,75-2,23)$ & 0,358 \\
$\begin{array}{l}\text { Tratamiento intensivo } \\
\text { (Hyper-CVAD) }\end{array}$ & 1,12 & $(0,40-3,13)$ & 0,826 \\
Riesgo alto & 1,65 & $(0,89-3,06)$ & 0,114 \\
\hline
\end{tabular}

\section{DISCUSIÓN}

El motivo del uso de Hyper-CVAD como régimen de primera línea se fundamentó en la hipótesis de que un régimen terapéutico más intensivo podría mejorar los resultados obtenidos hasta el momento con el protocolo institucional. La suspensión del enrolamiento fue debida, en su mayoría, a los eventos adversos serios como neutropenia febril, al igual que las muertes no asociadas a recaídas fueron mayores que el régimen institucional. A pesar de que no se realizó un estudio de costo, el régimen Hyper-CVAD es más costoso debido a un mayor requerimiento de antibióticos como al uso de estimulantes de colonias de granulocitos. Otro punto que se consideró hasta el momento del análisis fue la calidad de vida, ya que debido a las estancias prolongadas, los eventos adversos y el desgaste económico familiar, disminuyó significativamente, obligando a los pacientes a solicitar cambio de régimen a uno menos intensivo y económico.

Al igual que en México la opinión sobre la eficacia de HyperCVAD como primera línea aún está dividida. Buyukasik et al. en Turquía, lo compararon con su régimen estándar (CALGB-8811) y, a pesar de que la tasa de remisiones completas fueron aceptables $(84,2 \%)$, la mortalidad no asociada a recaída fue mayor $(29,7 \% \text { frente a } 5,9 \%)^{(13)}$. Lu et al. en China, compararon también su esquema estándar de dosis altas de AraC plus mitoxantrona frente a HyperCVAD y semejante a este reporte, a pesar de que no existieron diferencias significativas, la tasa de remisiones completas fue menor en el grupo de Hyper-CVAD (76\% frente a $87 \%)^{(14)}$. Morris et al. en Australia, en donde a pesar de ser un régimen tolerable y óptimo previo a un trasplante de progenitores hematopoyéticos, la toxicidad fue considerable obligando al cese del esquema en $29 \%$ de los casos que completaron una remisión completa ${ }^{(15)}$

En general, la mayoría de los autores coinciden que la principal limitante es la elevada toxicidad. Gills et al. analizaron este punto en 125 pacientes tratados con Hyper-CVAD, a 3 meses postratamiento, las citopenias persistieron en un $59 \%$ y normalizándose hasta los 9 meses, con un rango de 6 a 12 meses ${ }^{(16)}$. En dicho estudio 4 de los 125 pacientes desarrollaron una leucemia aguda o síndrome mielodisplásico secundario lo que orienta a un daño directo del régimen sobre las células progenitoras hematopoyéticas. Keane et al. describieron también este efecto, ya la toxicidad combinada de los dos brazos limitó la capacidad para poder movilizar progenitores hematopoyéticos ${ }^{(17)}$.

En México, la única experiencia disponible del uso de Hyper-CVAD como primera línea es la publicada por Arteaga et al. en el Instituto Nacional de la Nutrición Salvador Zubirán, y a diferencia de este reporte, se 
registraron mayores tasas de remisiones completas con el régimen Hyper-CVAD (85\%) en comparación con su régimen institucional 0195 (74\%) con una supervivencia global promedio de 15 meses ${ }^{(18)}$.

Se concluye que la decisión del uso de este régimen fuera de la Unión Americana se basa principalmente en la experiencia de cada centro, al no tratarse este estudio de un ensayo clínico aleatorizado no se puede brindar una recomendación sólida para restringir su uso, pero acorde a los resultados hallados se sugiere solo limitarlo a aquellas leucemias refractarias o en recaída.
Contribuciones de autoría: CORP, ACG, CB, HCS, participaron en la concepción del estudio, recolección de resultados y análisis, CORP, CB, HCS y EMF participaron en la redacción del artículo, CORP, ACG, ERF, JJKL, HCS, AMT, IOC, ASS y JCJ participaron en la revisión crítica y aprobación de la versión final del artículo, CORP, ACG, GLG, CB, JJKI, HCS, EMF, AMT, IOC participaron en el aporte de pacientes o material de estudio, CORP, ERF y HCS participaron en la obtención de financiamiento.

Conflictos de interés: los autores declaran no tener conflictos de interés

Financiamiento: autofinanciado

\section{REFERENCIAS BIBLIOGRÁFICAS}

1. Hoelzer D. Targeted therapy with monoclonal antibodies in acute lymphoblastic leukiemia. Curr Opin Oncol. 2013 Nov;25(6):701-6. doi: 10.1097/CCO.0000000000000009.

2. Zhao Y, Huang H, Wei G. Novel agents and biomarkers for acute lymphoid leukemia. J Hematol Oncol. 2013 Jun 18;6:40. doi: 10.1186/1756-8722-6-40.

3. Lukenbill J, Advani AS. The treatment of adolescents and young adults with acute lymphoblastic leukemia. Curr Hematol Malig Rep. 2013 Jun;8(2):91-7. doi: 10.1007/s11899-013-0159-0.

4. García-Manero G, Kantarjian HM. The hyper-CVAD regimen in adult acute lymphocytic leukemia. Hematol Oncol Clin North Am. 2000 Dec;14(6):1381-96.

5. Koller CA, Kantarjian HM, Thomas D, O'Brien S, Rios MB, Kornblau $S$, et al. The hyper-CVAD regimen improves outcome in relapsed acute lymphoblastic leukemia. Leukemia. 1997 Dec;11(12):2039-44.

6. Thomas DA, Faderl S, O'Brien S, BuesoRamos C, Cortes J, Garcia-Manero G, et al. Chemoimmunotherapy with hyperCVAD plus rituximab for the treatment of adult Burkitt and Burkitt-type lymphoma or acute lymphoblastic leukemia. Cancer. 2006 Apr 1;106(7):1569-80.

7. Ravandi F, O'Brien S, Thomas D, Faderl $S$, Jones D, Garris R, et al. First report of phase 2 study of dasatinib with hyperCVAD for the frontline treatment of patients with Philadelphia chromosomepositive $(\mathrm{Ph}+)$ acute lymphoblastic leukemia. Blood. 2010 Sep 23;116(12):20707. doi: 10.1182/blood-2009-12-261586.

8. Kantarjian H, Thomas D, O'Brien S, Cortes J, Giles F, Jeha S, et al. Long-term follow-up results of hyperfractionated cy- clophosphamide, vincristine, doxorubicin, and dexamethasone (Hyper-CVAD), a dose-intensive regimen, in adult acute lymphocytic leukemia. Cancer. 2004 Dec 15;101(12):2788-801.

9. Xu W, Li JY, Qian SX, Wu HX, Lu H, Chen LJ, et al. Outcome of treatment with Hyper-CVAD regimen in Chinese patients with acute lymphocytic leukemia. Leuk Res. 2008 Jun;32(6):930-5.

10. Combariza JF, Casas CP, Rodrpiguez M, Cardona AF, Ospina E, Grajales M. Supervivencia en adultos con leucemia linfoide aguda de novo tratados con el esquema HyperCVAD en el Instituto Nacional de Cancerología (Colombia), entre enero de 2001 y junio de 2005 . Rev Colom Cancerol. 2007;11(2):92-100.

11. Annino L, Vegna ML, Camera A, Specchia G, Visani G, Fioritoni G, et al. Treatment of adult acute lymphoblastic leukemia (ALL): long-term follow-up of the GIMEMA ALL 0288 randomized study. Blood. 2002 Feb 1;99(3):863-71.

12. Ramos C, Rozen E, León M, Martínez T A, Olarte I, Catellanos $\mathrm{H}$, et al. Tratamiento de la leucemia linfoide aguda del adulto. Experiencia de un hospital en la Ciudad de México. Rev Med Chil. 2011 Sep;139(9):1135-42. doi: /S003498872011000900004.

13. Buyukasik Y, Acar K, Kelkitli E, Uz B, Serefhanoglu S, Ozdemir E, et al. HyperCVAD régimen in routine management of adult acute lymphoblastic leukemia: a retrospective multicenter study. Acta Haematol. 2013;130(3):199-205. doi: $10.1159 / 000351172$.

14. Lu Q, Jeong W, Katragadda S, Seiter K, Shi Q, Liu D. High-dose cytarabine-mitoxantrone versus Hyper-CVAD in adult acute lymphoblastic leukemia and burkitt's lym- phoma: a single center experience of two induction regimens. Open Leukemia J. 2009;2(1):1-6.

15. Morris K, Weston $H$, Mollee P, Marlton P, Gill D, Kennedy G. Outcome of treatment of adult acute lymphoblastic leukemia with hyperfractionated cyclophosphamide, doxorubicin, vincristine, dexamethasone/methotrexate, cytarabine: results from an Australian population. Leuk Lymphoma. 2011 Jan;52(1):85-91. doi: $10.3109 / 10428194.2010 .532889$.

16. Gill S, Lane SW, Crawford J, Cull G, Joske D, Marlton P, et al. Prolonged haematological toxicity from the hyper-CVAD regimen: manifestations, frequency, and natural history in a cohort of 125 consecutive patients. Ann Hematol. 2008 Sep;87(9):727-34. doi: 10.1007/s00277-008-0488-6.

17. Keane C, Gibbs S, Seymour JF, Mills AK, Grimmett K, Van Kuilenberg R, et al. The Hyper-CVAD chemotherapy regimen has an adverse long-term impact on the ability to mobilize peripheral blood stem cells, which can be readily circumvented by using the early cycles for mobilization. Hematol Oncol. 2006 Sep;24(3):159-63.

18. Arteaga-Ortiz L, Buitrón-Santiago N, Rosas-López A, Rosas-Arzate G, Armengolt-Jiménez A, Aguayo A, et al. Experiencia del INCMNSZ en pacientes adultos con leucemia linfoide aguda. Cohorte 2003-2007 con esquemas de tratamiento Hiper-CVAD y Protocolo 0195. Rev Invest Clin. 2008;60(6):459-69.

Correspondencia: Christian Omar Ramos Peñafiel Dirección: Camino a Chapultepec 2C,

Cofradia de San Miguel Cuautitlán Izcalli

Estado de México, CP 54740. México.

Teléfono: 5523351588

Correo electrónico:leukemiachop@hotmail.com 